\title{
Adaptation of Reminiscence Functions Scale for Bangladeshi Population
}

\author{
Asheek Mohammad Shimul*, Jannatul Ferdous \\ Department of Psychology, University of Dhaka, Bangladesh
}

Copyright $(\odot 2016$ by authors, all rights reserved. Authors agree that this article remains permanently open access under the terms of the Creative Commons Attribution License 4.0 International License

\begin{abstract}
Objective of the present study was to adapt the Reminiscence Functions Scale (RFS) in Bangla to be used in the context of Bangladeshi culture and background. Data were collected from 300 adult participants using survey method. Among the participants 171 were male and 129 were female. Their age range was 17 to 90 (Mean $=37.69$, $\mathrm{SD}=12.47$ ). Psychometric analysis included item analysis, principal component analysis with varimax rotation, test-retest reliability and internal consistency reliability using Cronbach's alpha. The 43-items were found to be psychometrically acceptable to construct this Bangla version of RFS by Webster [9]. Data revealed eight factors, namely -Boredom Reduction, Death Preparation, Identity, Problem-Solving, Conversation, Intimacy Maintenance, Bitterness Revival, and Teach/Inform. Test-retest and internal consistency reliability of the instrument was found .81 and .89 respectively. Psychometric evidences indicate this self-report Likert instrument is both reliable as well as valid measure of reminiscence function and hence suggested to be usable in the context of Bangladesh.
\end{abstract}

Keywords Reminiscence, Adaptation, Principal Component Analysis, Psychometrics

\section{Introduction}

Reminiscence, a process of recalling memories from our personal past, is thought to be a universal phenomenon across the world. Researchers in this field paid their attention to different aspects of reminiscence, someone focused on process [5], whereas someone talks about contents [11]. Most of the people, irrespective of culture and language, think about the past at different points throughout their lives. Sometimes they reminisce deliberately, and generatively, whereas sometimes people reminisce unintentionally or spontaneously, sometimes privately or sometimes with other people, and it might be involve remembering both happy and sad episodes of their daily life.

Different types of reminiscence have been found in memory literature, some are different and some are overlapping. Coleman [1] distinguished between simple reminiscence and informative reminiscence whereas LoGerfo [4] added evaluated life review and obsessive reminiscence with informative reminiscence. On the other hand using content analysis Wong \& Watt [11] identified six types of reminiscence named as Integrative reminiscence, Instrumental reminiscence, Transmissive reminiscence, Obsessive reminiscence, Narrative reminiscence, and Escapist reminiscence (See [11] for review). Some of these types are not mutually exclusive since reminiscence may serve several purposes at the same time. Webster \& Haight [10] reviewed the systems proposed by various authors for categorizing functions of reminiscence. They identified significant overlap among the classifications and they proposed the taxonomy with eight distinct purposes of reminiscence. Reminiscence can be used to discover meaning and continuity (Identity), to draw on past experience to solve present problems and to cope (Problem-Solving), to provide an instructive story (Teach/Inform), to give substance for telling a descriptive story (Conversation), to dwell on the past and escape from the present (Boredom Reduction), to obsess about unresolved disturbing past events (Bitterness Revival), to prepare for death (Death Preparation), and to maintain alive memories of significant others (Intimacy Maintenance).

Description of the original scale: Reminiscence Functions Scale (RFS) by Webster [9] is a 43 items questionnaire with 6 point Likert type of scale which is extensively used to assess reminiscence function over the life course. It has 7 factors with good reliability measured through internal consistency ranged from .79 to .89 . This instrument has higher level of predictive validity which was assessed by both personality traits and developmental aspects.

The current study attempted to adapt the RFS in Bangla language and culture. This study is important because the findings from using this instrument can be used both for clinical and non-clinical purposes. This RFS scale can be useful tool in clinical setting understanding people having mild level of clinical psychological disturbance such as anxiety, depression, and disruptive behavior symptoms. Also, 
the RFS scale may be administered in combination for more comprehensive assessment and profiling or in specific combinations tailored to specific needs of the target oriented service recipients. One of the most common stumbling blocks to integration of existing evidence regarding reminiscence is the paucity of psychometrically sound instruments for assessing various purposes of memory retrieval. In order for meaningful comparisons between research projects to occur, clinical and experimental investigators need a readily available, psychometrically sound instrument that produces comparable scores across studies. To my knowledge there is no available instrument to understand memory functions especially for Bangladeshi population. Therefore, the present study is designed to adapt the RFS for use in Bangladesh.

An adapted instrument enables the researcher to make comparisons in the data from different samples and from different backgrounds, which leads to greater fairness in the evaluation and enables a greater ability to generalize and also helps one to investigate differences within an increasingly diverse population $[3,7]$. The steps of adaptation of an instrument intended to be used for measuring psychological constructs requires some very careful planning in process. Instruments barely translated to any language are absolutely insufficient to capture the construct because of psychometrical demand in science. As we know that, the terms "adaptation" and "Translation" are distinct. The former includes all the processes concerning the cultural fit of the instrument beyond mere translation. On the other hand, translation is merely the first stage of the adaptation process. It should be considered about the cultural, idiomatic, linguistic and contextual aspects concerning its translation when adapting an instrument.

\section{Method}

Consulting the guidelines of International Test Commission (2005), six steps were followed in the adaptation process of Bangla RFS.

Step one: Confirmation of same construct: Available literature like scientific journal articles, periodicals, books, and culturally adapted scales published on reminiscence functions were consulted to determine whether the constructs of the original reminiscence function scale convey the same meanings for Bangladeshi people and their culture. To achieve this construct equivalence, panels of experts (who are familiar on memory reminiscence and test adaptation) were involved. They judge each and every items of the scale and ensure its constructs similarity. Binding all of their judgments, a consensus was found which convey that the construct under the current study really do exist. The experts also recommend that the same definition could be equally applicable to language and cultural group of Bangladesh.

Step two: Forward translation: Two professional translators, who graduated from English department of Dhaka University, were selected for translating the original
RFS from English to Bangla, and they were absolutely blind about who the other person is doing translation. Two criteria were followed before selection of the translators, 1) their mother tongue has to be Bangla, and 2) they have to be familiar with American language, literature, and culture. Without consulting one another they independently translated the English version of the RFS to Bangla. Both of the translators put much iterative effort in selecting the best words, expressions, or items to translate their respective translated versions. Thus, the preliminary Bangla version inventory was prepared. A professor of Linguistics from University of Dhaka was then consulted to check for the language and sentence structure and quality of translation, and conceptual equivalence of words or phrases, but not a word-for-word translation. Following the expert's opinion, some modifications were done which uphold the items' expression, suitability to measure the construct in Bangladeshi culture.

Step three: Back translation: Again three professional translators were recruited who without consulting one another independently translated the Bangla version of the RFS to English. The translators' mother tongue was Bangla, but their medium of education was English. As a result, all of them belong to a good command in both English and Bangla language and they were assigned to the task of translating the Bangla version into English. A panel consisting of three members having expertise in psychometrics and proficiency in English judged the equivalence of the original English version and the back-translated version of the RFS. High degree of consensus among the panel members were observed and the back-translated version of the RFS was found to be very much like the original one which indicating the correctness of forward translation. The Bangla version was then considered as ready to use for next processes.

Step four: Pre-testing and cognitive interviewing: Before administering the finalized Bangla version of RFS to a large group of sample a pre-test was carried out on a group of 30 convenient samples which included male and female (age range 17 to 90 years) having rural urban composition. Objectives and significance were conveyed to the participants before they took part in this study. Participants who showed their interest were selected as participants. To have primary validity evidence for each of the items of RFS, three methods were employed namely, the scale administration, semi-structured interview and item analysis.

Scale administration-In this part of the study translated Bangla version of RFS was administered to the respondents in convenient locations. A group of six trained data collectors were employed to administer this scale. All of them were trained well before the administration. At first, informed consents were taken and then participants were asked to read the instructions on the top of the RFS questionnaire very carefully. Then participants were also orally instructed to make it clear what they have to do here. They were made assured that the information collected from them would be kept strictly confidential and would be used only for the research purpose. The questionnaire was 
administered individually to each participant. They were instructed to answer every question honestly and to select only one response for each question. Participant's responses to each item indicate how frequently the statement was true for them. They were instructed that if their answer is NEVER for any statement of the questionnaire they have to rate that statement as 1, If their answer is RARELY rate as 2, SELDOM as 3, OCCASIONALLY is as 4, OFTEN as 5 , and if their answer is VERY FREQUENTLY they have to rate the statement as 6 where there is nothing as right or wrong answer here. They were told to answer as truthfully as possible and work relatively quickly. During the questionnaire administration, the participants were allowed to ask questions about words or concepts which they did not understand. Then the words, expressions or sentences that the participants asked about were noted to check whether it was necessary to modify them. Approximately 15-20 minutes were taken by the participants to complete their task.

Interview- To find out whether any word, concept, or expression that needs to be addressed further because of its difficulty, confusion, unacceptableness or offensiveness, the present study used person to person interview method. If there is anything that participants do not understand or confusing, they were asked to put their alternatives or they were asked "how would you ask the question if you were prepare this item?" Through this procedure contextualization of their language was make ensure.

Item analysis-After the completion of administering the scale, participant's responses in each item were analyzed to determine corrected item-to-total correlation which indicated the appropriateness of each item. To sort it out which items are not able to measure what the whole scale is supposed to measure, item to total score correlation was calculated. And items which show low or negative correlation were paid extra attention and modified. Item analyses for this questionnaire revealed that a total of 10 items had low positive correlation and 10 items had negative correlation with total score. Interestingly, these were the items which the respondents found confusing and difficult. However, internal consistency reliability (alpha coefficient) for each factor was good ranging from .49 to .72 . The panel members who worked in the back translation phase as judges had modified the language of the faulty items in consensuses with each other which resulted in the revised preliminary Bangla version of Reminiscence Functions Scale.

Step five: Pre-testing II: Again following two methods namely scale administration and item analysis, the revised preliminary Bangla version of RFS was administered on couple of respondents as second pre-testing.

Scale administration- A total of 44 samples (age range $21-68 ; 46 \%$ male and $54 \%$ female; $30 \%$ rural and $70 \%$ urban) were selected purposively following convenient sampling method to administer the whole scale. Same procedure like the first pre-testing was followed this time for scale administration and data collection.
Item analysis - Item analysis in the second pre-testing showed a marked improvement from statistical as well as conceptual framing. Internal consistency of the whole scale ranged from .69 to .89 which better in comparison to first pre-testing. On the other hand, satisfactory levels of corrected item-to-total correlation coefficients were observed for almost all the items except for two items. These items were then revised once again through expert opinion and included in the main scale and then retained for field test.

Step six: Field test: Being confirmed from two times of the pre-tests, now the Bangla version of RFS is absolutely applicable for the Bangladeshi population, a field test was carried out to determine reliability and validity of the Bangla version Reminiscence Functions Scale. Purposive and convenience sampling technique was used to collect data from 313 (after data cleaning finally 300 respondents retained for data analysis) adults aged between 17 and 79 years. All of the 300 participants comprised of two groups age ranging from 17 to 39 and 40 to 40 up. First group comprised of 169 participants (90 male and 79 female, 102 urban resident and 67 rural), and the second group had 131 participants (81male and 50 female, 99 urban resident and 32 rural). Following an incidental sampling method data were collected from the students (29\%), employees (32\%), businessmen (26\%), and housewives (13\%) who were available and willing to participate in the study. Although nature of the sampling was incidental but two inclusion criteria were applied during sample selection: (a) completed secondary school education, and (b) had no severe physical or mental condition that might interfere with the assessment.

\section{Results}

At first the result of the study was analyzed by using Principal Components Analysis with varimax rotation as theoretically factors were presumed to be unrelated. In our present study the adoption of item and factor retention was made on the basis of some criteria. That is, only those items that must had a minimum Eigen value of 1, minimal item loading on a factor was equal to $\geq .50$, and at least 4 or more items meeting the criteria (loading $\geq .50$ ) had been considered for a factor. It was done to facilitate reliability checks. Maintaining the above criteria and rotating the loadings to confirmed eight clearly distinct factors corresponding to the original RFS, labeled as follows: (1) Boredom Reduction, (2) Death Preparation, (3) Identity, (4) Problem-Solving, (5) Conversation, (6) Intimacy Maintenance, (7) Bitterness Revival, and (8) Teach/Inform. A total of 43 of items were retained in the analysis. Table 1 shows the factor loadings statistics for adapted Bangla version of Reminiscence Functions Scale on each factor for all 43 RFS questions. Sample adequacy was determined examining KMO index and Bartlett's test of spheicity reported as .92 and 1815.4536 , $p<.0001$ (Strickland [6]). 
Table 1. Factor loading for adapted Bangla version of Reminiscence Function Scale

\begin{tabular}{|c|c|c|c|c|c|c|c|c|}
\hline \multirow{2}{*}{ RFS items } & \multicolumn{8}{|c|}{ Factor loadings } \\
\hline & 1 & 2 & 3 & 4 & 5 & 6 & 7 & 8 \\
\hline \multicolumn{9}{|l|}{ Boredom } \\
\hline Reduction & .79 & & & & & & & \\
\hline Item 3 & .63 & & & & & & & \\
\hline Item 11 & .57 & & & & & & & \\
\hline Item 16 & .56 & & & & & & & \\
\hline Item 19 & .56 & & & & & & & \\
\hline Item 21 & .53 & & & & & & & \\
\hline \multicolumn{9}{|l|}{ Item 37} \\
\hline \multicolumn{9}{|l|}{ Death } \\
\hline Preparation & & .81 & & & & & & \\
\hline Item 2 & & .71 & & & & & & \\
\hline Item 9 & & .64 & & & & & & \\
\hline Item 29 & & .58 & & & & & & \\
\hline Item 33 & & .51 & & & & & & \\
\hline Item 35 & & .50 & & & & & & \\
\hline \multicolumn{9}{|l|}{ Item 38} \\
\hline \multicolumn{9}{|l|}{ Identity } \\
\hline Item 8 & & & .77 & & & & & \\
\hline Item 10 & & & .70 & & & & & \\
\hline Item 24 & & & .62 & & & & & \\
\hline Item 26 & & & .61 & & & & & \\
\hline Item 32 & & & .57 & & & & & \\
\hline Item 36 & & & .55 & & & & & \\
\hline \multicolumn{9}{|l|}{ Problem } \\
\hline solving & & & & .80 & & & & \\
\hline Item 4 & & & & .65 & & & & \\
\hline Item 12 & & & & .56 & & & & \\
\hline Item 18 & & & & .55 & & & & \\
\hline Item 31 & & & & .51 & & & & \\
\hline Item 39 & & & & .50 & & & & \\
\hline \multicolumn{9}{|l|}{ Item 42} \\
\hline \multicolumn{9}{|l|}{ Conversation } \\
\hline Item 6 & & & & & .76 & & & \\
\hline Item 7 & & & & & .72 & & & \\
\hline Item 22 & & & & & .71 & & & \\
\hline Item 28 & & & & & .70 & & & \\
\hline Item 34 & & & & & .53 & & & \\
\hline \multicolumn{9}{|l|}{$\begin{array}{l}\text { Item } 34 \\
\text { Intimacy }\end{array}$} \\
\hline Item 5 & & & & & & .79 & & \\
\hline Item 14 & & & & & & .62 & & \\
\hline Item 25 & & & & & & .61 & & \\
\hline Item 41 & & & & & & .60 & & \\
\hline \multicolumn{9}{|l|}{$\begin{array}{c}\text { Item 41 } \\
\text { Bitterness }\end{array}$} \\
\hline Item 13 & & & & & & & .78 & \\
\hline Item 15 & & & & & & & .70 & \\
\hline Item 17 & & & & & & & .61 & \\
\hline Item 40 & & & & & & & .58 & \\
\hline Item 43 & & & & & & & .55 & \\
\hline \multicolumn{9}{|l|}{$\begin{array}{l}\text { Item } 43 \\
\text { Teaching }\end{array}$} \\
\hline Item 1 & & & & & & & & .74 \\
\hline Item 20 & & & & & & & & .72 \\
\hline Item 23 & & & & & & & & .70 \\
\hline Item 27 & & & & & & & & .61 \\
\hline Item 30 & & & & & & & & .56 \\
\hline Eigenvalue & 8.16 & 3.17 & 2.42 & 2.26 & 2.08 & 1.86 & 1.62 & 1.46 \\
\hline$\%$ variance & 19.42 & 7.56 & 5.76 & 5.38 & 4.96 & 4.44 & 3.86 & 3.48 \\
\hline $\begin{array}{l}\text { \%cumm. } \\
\text { Vaiance }\end{array}$ & 19.42 & 26.97 & 32.74 & 38.12 & 43.08 & 47.52 & 51.38 & 54.86 \\
\hline
\end{tabular}


Table 2 indicated that among the eight factors there were three exceptions. That is, no significant relationship had been found between factor (5) Conversation with factor (2) Death Preparation and factor (7) Bitterness Revival. Same trend no significant relationship was observed with factor (4) Problem Solving (8) Teach or Inform.

Table 2. Factor intercorrelation matrix

\begin{tabular}{ccccccccc}
\hline Factors & \multicolumn{7}{c}{ Factors } \\
\hline & $\mathbf{1}$ & $\mathbf{2}$ & $\mathbf{3}$ & $\mathbf{4}$ & $\mathbf{5}$ & $\mathbf{6}$ & $\mathbf{7}$ & $\mathbf{8}$ \\
$\mathbf{1}$ & - & .41 & .32 & .31 & .22 & .33 & .29 & .33 \\
$\mathbf{2}$ & & - & .29 & .35 & $.29 *$ & .28 & .27 & .36 \\
$\mathbf{3}$ & & & - & 41 & .31 & .35 & .31 & .52 \\
$\mathbf{4}$ & & & & - & .25 & .29 & .54 & $.29 *$ \\
$\mathbf{5}$ & & & & & - & .25 & $.21^{*}$ & .31 \\
$\mathbf{6}$ & & & & & & - & .30 & .28 \\
$\mathbf{7}$ & & & & & & & - & .33 \\
$\mathbf{8}$ & & & & & & & & \\
\hline
\end{tabular}

* No significant relationship

As a means of assessing factor reliability of the adapted RFS in Bangla, internal consistency scores were computed using coefficient alpha and which had been found as 0.89 .

Table 3 indicates that in our present research factor's internal consistency was good ranging from .64 for factor 6 (Intimacy maintenance) to .83for factor 2(Death Preparation).

Table 3. Internal consistency of eight factors of Bangla version of RFS

\begin{tabular}{ccccccccc}
\hline & \multicolumn{8}{c}{ Factors } \\
\cline { 2 - 9 } & $\mathbf{1}$ & $\mathbf{2}$ & $\mathbf{3}$ & $\mathbf{4}$ & $\mathbf{5}$ & $\mathbf{6}$ & $\mathbf{7}$ & $\mathbf{8}$ \\
\hline $\begin{array}{c}\text { Alpha } \\
\text { value }(\alpha)\end{array}$ & .81 & .83 & .79 & .78 & .71 & .64 & .68 & .67 \\
\hline
\end{tabular}

To determine the test-retest reliability of this adapted Bangla version of RFS, 44 participants (those who were used in pretesting II stage) were once again administered the scale with a gap of 21 days. Cronbach's alpha for 43 items of RFS was found .81. This Bangla version of RFS has been found to similar with original version (Webster, [9]) where there was no gender difference. In this present study t-test between male and female participants were compared and no statistical difference had been observed $(t=0.064, p>.05)$.

\section{Discussion}

The purpose of the present study was to adapt the Reminiscence Functions Scale (RFS) into Bangla for using in the context of Bangladeshi culture and background. The adapted Bangla version of RFS has been found to be reliable, valid measure of many fundamental uses of reminiscence in adulthood, especially for Bangladeshi population. It has been found to be consistent with findings of Wong \& Watt's [11] taxonomy. RFS is a 43 items 6-point scale comprised of eight factors labeled as-Boredom Reduction, Death Preparation, Identity, Problem Solving, Conversation, Intimacy maintenance, Bitterness Revival, and Teach/Inform. After checking by experts at first a pretest was carried out for the adapted Bangla RFS on 30 adults including both male and female age ranging from 17-90 years old. Internal consistency reliability (alpha coefficient) for each factor was good ranging from .49 to .72 . The internal consistency and inter-item correlation indicated that the instrument is reliable and all items of the questionnaire are important. Being confirmed from the pre-tests that the Bangla version RFS is applicable for the Bangladeshi population. A field test was carried out to determine reliability and validity of the Bangla version RFS Purposive and convenience sampling techniques were used to collect data from 300 adults.

Result of the present study was analyzed by using Principal Components Analysis. A total of 43 of items were retained in the analysis. Results presented in Table 1 shows the factor loadings statistics for adapted Bangla version of Reminiscence Functions Scale on each factor for all 43 RFS questions. Here it was found that all the factors contained at least four items meeting the criteria adopted for item and factor retention. Results presented in Table 2 indicated that among the eight factors there were three major exceptions, among those there was no significant relationship. Here we can see that, there is no significant relationship with factor (2) Death Preparation to factor (5) Conversation, factor (4) to factor (8) Teach or Inform, and factor (5) Conversation to factor (7) Bitterness Revival. Results presented in Table 3 indicates that in our present research factor's internal consistency was good ranging from .64 for factor 6 (Intimacy maintenance) to .83 for factor 2 (Death Preparation). And lastly Table 3 indicates the reliability statistics of our present study. Seeing table 3 we can say that the Cranbach's Alpha of the study was .892 which is very good.

From the above discussion we saw that both internal consistency and test retest reliabilities of our present study were highly satisfactory. Significant correlations within the factors provided the evidence for construct validity. Thus we can confidently say that the adapted Bangla version of RFS is psychometrically sound and culturally appropriate. Therefore, researchers working with memory can confidently use the instrument on Bangladeshi population for a variety of purposes.

The findings of the present study are quite comparable to previous studies related to it. That is, in our present study the Internal Consistency for the eight factors has been found ranging from .69 to .89 where in the study of Webster [9] it was found ranging from .79 to .89 ; in the study of Daniela, Guedes, Antonio, Martin [2] it was found ranging from .82 to .88 for five factors. Internal consistency of the subscale of RFS is sound in comparison to Washington [9]. From the overall discussion it is clear that the adapted Bangla Reminiscence Functions Scale is completely applicable and suitable for further use in the context and background of Bangladeshi culture.

One of the mentionable limitations of the study is use of a 
convenience sample. Inclusion of ethnic population in Bangladesh would be important insight in this context.

\section{REFERENCES}

[1] Coleman PG. Measuring reminiscence characteristics from conversation as adaptive features of old age. International Journal of Aging and Human Development, 1974; 5, 281-294.

[2] Daniela CG, Guedes JM, Antonio MF, Martin I. Psychometric properties functions scale for the Portuguese population: A preliminary report. International Journals of Aging and Human Development. 2010. 71 (2) 153-166.

[3] Hambleton RK. Issues, designs, and technical guidelines for adapting tests into multiple languages and cultures. In R. K. Hambleton, P. F. Merenda, \& C. D. Spielberger (Eds.), Adapting educational and psychological tests for cross-cultural assessment (pp. 3-38). Mahwah, NJ: Lawrence Erlbaum. 2005.

[4] LoGerfo M. Three ways of reminiscence in theory and practice. International Journal of Aging and Human Development, 1980-81; 12, 39-48.
[5] Parker, R. G. (1995). Reminiscence: A continuity theory framework. The Gerontologist, 35, 515-525.

[6] Strickland O. Using factor analysis for validity assessment: Practical considerations (Editorial). Journal of Nursing Measurement, 2003; 11(3). 203-205.

[7] Vivas E. Estudios transculturales: Una perspectiva desde los trastornos alimentarios. In S. M. Wechsler \& R. S. L. Guzzo (Orgs.),Avaliação psicológica: Perspectiva internacional (2a ed., pp. 463-481). São Paulo: Casa do Psicólogo. 1999.

[8] Washington G. Modification and psychometric testing of the reminiscence function scale. Journal of Nursing Measurement. 2009; 17 (2), 134-147.

[9] Webster JD. Construction and Validation of the Reminiscence functions Scale. Journal of Gerontology: Psychological Sciences. 1993; 48, 256-262.

[10] Webster JD, Haight BK. Memory lane milestones: Progress in reminiscence definition and classification. In B. K. Haight \& J. D. Webster (Eds.), The art and science of reminiscing: Theory, research, methods, and applications. Washington, DC: Taylor and Francis. 1995.

[11] Wong PTP, Watt LM. What types of reminiscence are associated with successful aging? Psychology and Aging. $1991 ; 6,272-279$. 